\title{
Design and Simulation of Solid Polymer Micro Needles Array
}

\author{
Samir S. Abubaker, Zhang Yajun, Dong Liqun \\ College of Mechanical and Electrical Engineering, Beijing University of Chemical Technology, \\ Beijing, China.
}

Samir@mail.buct.edu.cn, aghaydi@gmail.com, zhyj@mail.buct.edu.cn, qonglq@mail.buct.edu.cn*

Keywords: Polymer micro-needle, Mechanical behavior, Bending and Buckling forces.

Abstract. Microneedles have several applications in beauty and medicine. Many researches are investigating the manufacturing of microneedles on a mass-production scale with relatively low costs, relatively few studies on the mechanical behavior of the microneedle into skin and piercing manner, especially few about polymer microneedles. Microneedles have been shown to greatly increase the skin's permeability, allowing for an effective transfer of drugs. They however still face numerous challenges. In this paper mechanical behavior of the micro needle was simulated by MATLAB and ANSYS via the results of suffered and strength analysis.

\section{Introduction}

With the development of micro-electro-mechanical systems (MEMS) and materials science, a more efficient and safer traditional transdermal drug delivery (TDD) like microneedle (MN) emerges [1]. MN technology has received much attention in the medical community and the beauty industry. Microneedles (MNs) can eliminate the side effects of typical injection needle, such as skin damage, infection, and pain [2], and easy to use, in contrast to hypodermic needles [3]. Furthermore as an alternative, transdermal patches provide convenient, time-release delivery that avoids the gastrointestinal tract [4]. Appeared clearly for MNs technology development, it becomes possible for MNs array to strengthen the TDD, in addition to the increase of the skin permeability, therefore to deliver drugs into skin, such as insulin and vaccine [5], providing a new direction for drug delivery systems. MNs will play a decisive role in promoting micro-sample analysis, trace injection, blood fluid analysis, sampling and vaccines against influenza. MNs have been demonstrated to be pain-free and potentially low-cost and easy-to use [6]. Generally, MNs should not break when bent slightly, and not rupture when pulled out after piercing the skin (except dissolvable MN).

In this paper, solid polymer MNs was designed, a mechanical model of MN was established, and then an appropriate force equations was built. After the strength of a polymer $\mathrm{MN}$ was verified, the piercing situations of different shapes were simulated, the optimized structures of MNs were obtained. Based on the stress condition, three kinds of common polymers with different geometries were simulated using MATLAB and ANSYS. The results showed that all kinds could satisfy the strength requirements. Based on that, PMMA, PC and PLA were chosen to fabricate MNs.

\section{Optimization design of MN}

The mechanical model is simply conical frustum model for solid $\mathrm{MN}$, it is mainly under a bending force $\left(F_{b}\right)$ perpendicular to the needle that makes MN bending laterally, and a compression force $\left(F_{p}\right)$ parallel to the needle that makes MN compression axially and buckling. The mechanical model of $\mathrm{MN}$ is shown in Fig. 1 below. 


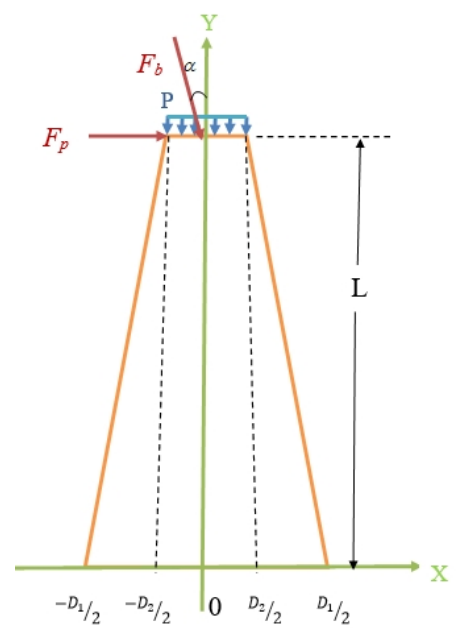

Fig. 1. Mechanical model of MN

$D_{1}$ and $D_{2}$ are the base and tip diameter of frustum $\mathrm{MN}$ respectively, $L$ is the length of $\mathrm{MN}$. A limited condition is $D_{1}>2 D_{2}$. To simplify calculations, a linear mathematical equation is established on the boundary condition of $\mathrm{MN}$.

$$
y=\left(\frac{L}{D_{1}-D_{2}}\right)\left(-2 x+D_{1}\right)
$$

where $x \in\left[\frac{D_{2}}{2}, \frac{D_{1}}{2}\right], y \in[0, L]$.

\section{Bending and compression force analysis}

When penetrating the skin, because of the influence of bending force, MN's have relatively lateral movement when they penetrate the cuticle, which can be regarded as a cantilever beam suffered a lateral force [7], so the bending stress subjected to the MN can withstand is:

$$
\sigma_{1}=\frac{F_{b} l}{W}
$$

Where $\sigma_{l}$ is the $\mathrm{MN}$ bending strength that $\mathrm{MN}$ can withstand, $W$ is the bending section modulus for the MN circular gross sectional area (frustoconical) $W=\frac{\left(\pi d^{3}\right)}{32}$, and $l=L-y, \mathrm{~d}=2 x$, therfore Eq. 2 will be defined as follows:

$$
\sigma_{1}=\frac{F_{b}\left[L-\left(\frac{L}{D_{1}-D_{2}}\right)\left(-2 x+D_{1}\right)\right]}{\frac{\pi}{32}(2 x)^{3}}
$$

MNs penetrate the skin when subjected to the most important external forces, the axial compression forces (pressure force). Under the action of compression force, the stress $\sigma_{2} \mathrm{MN}$ can withstand is:

$$
\sigma_{2}=\frac{F_{p}}{A_{t}}
$$

Where $\sigma_{2}$ is the compressive strength that $\mathrm{MN}$ can withstand, $F_{P}$ is the pressure force (compression force) that $\mathrm{MN}$ can withstand, $A_{t}$ is the MN tip area.

\section{Force calculation method of MNs}

Due to the elasticity factor of the skin, bending force against MNs is very small. Straight plug in micro-needle array, located on the edge portion of the MNs prior to pierce the skin by a very small lateral force. Suppose the angle $\alpha$ exist between three groups MN array edge, MNs top surface and the 
skin, the force will be perpendicular to MNs contact surface, as shown in Fig.1. Theoretically, the pressure required to penetrate the human skin is 3.183MPa [8]. Based on the above analysis, the MN requires pressure to penetrate into the skin, so the pressure force $F_{P}$ needed is given as:

$$
F_{p}=3.183 \frac{\pi D_{2}^{2}}{4}
$$

From Eq. 5, the suffered compression force depends on the tip diameter of the needle. When the MN subjected to bending force, the weakest point is at the cross-sectional area of $(3 / 2) \mathrm{D}_{2}$. Therefore, the $\mathrm{MN}$ pierce the skin when it is subjected to lateral bending force, thus the bending force $F_{b}$ is defined as follows:

$$
F_{b}=\frac{1}{2} \sin \alpha\left(3.183 \frac{\pi D_{2}^{2}}{4}\right)
$$

While the maximum stress is defined as follows:

$$
\sigma_{\max }=\sigma_{1}+\sigma_{2} \leq[\sigma]
$$

Where $\sigma_{2}$ is $3.183 \mathrm{MPa}$, therefore $\sigma_{1}$ determines the critical cross section of the $\mathrm{MN}$ and its final stress value. Derivation of the above function can achieve the maximum value at $x=(3 / 4) D_{2}$, which is dangerous $\mathrm{MN}$ section, therefore the maximum stress is given as:

$$
\sigma_{\max }=\sigma_{2}\left[\frac{16 L}{27\left(D_{1}-D_{2}\right)} \sin 2 \alpha+1\right] \leq[\sigma] .
$$

Using Eq. $(6,8)$ to estimate the bending force and maximum strength for the mechanical model, thus determine the material can be used for this model. Table 1 shows some different MNs geometries with the calculated forces and stress piercing the skin with $30^{\circ}$ and $45^{\circ}$ bending force angel.

Table 1. Bending force and maximum stress for some different sizes of $\mathrm{MN}$

\begin{tabular}{lccccccc}
\hline Type & $\mathrm{L}$ & $\mathrm{D}_{1}$ & $\mathrm{D}_{2}$ & $\mathrm{~F}_{\mathrm{b}}\left(30^{\circ}\right) \mathrm{mN}$ & $\mathrm{F}_{\mathrm{b}}\left(45^{\circ} \mathrm{mN}\right.$ & $\sigma\left(30^{\circ}\right) \mathrm{MPa}$ & $\sigma\left(45^{\circ}\right) \mathrm{MPa}$ \\
\hline $\mathrm{A}$ & 200 & 150 & 30 & 1.0 & 1.1 & 6.0 & 6.3 \\
$\mathrm{~B}$ & 550 & 250 & 50 & 2.7 & 3.1 & 7.7 & 8.3 \\
$\mathrm{C}$ & 800 & 400 & 100 & 10.8 & 12.5 & 7.5 & 8.2 \\
\hline
\end{tabular}

Therefore, materials with yield strength above this value can withstand the forces needed to pierce the skin without breaking and buckling the needle.

\section{Stress and strain analysis (ANSYS) MN}

The research focused on the influences of MNs length and diameters on stress, strain and deformation.

\begin{tabular}{|c|c|c|c|c|c|c|c|c|c|c|}
\hline \multirow[t]{2}{*}{ Pos. } & \multirow[t]{2}{*}{ Static Structural } & \multicolumn{3}{|c|}{$\mathrm{PC}$} & \multicolumn{3}{|c|}{ PMMA } & \multicolumn{3}{|c|}{ PLA } \\
\hline & & A & B & $\mathrm{C}$ & A & B & $\mathrm{C}$ & A & B & $\mathrm{C}$ \\
\hline & Total def.(mm) & $6.2 \mathrm{e}^{-5}$ & $1.5 \mathrm{e}^{-4}$ & $2.8 \mathrm{e}^{-4}$ & $4.5 \mathrm{e}^{-5}$ & $1.2 \mathrm{e}^{-4}$ & $2.2 \mathrm{e}^{-4}$ & $3.7 \mathrm{e}^{-5}$ & $9.3 \mathrm{e}^{-5}$ & $2.1 \mathrm{e}^{-4}$ \\
\hline & Stress (MPa) & 2.8 & 2.9 & 2.9 & 2.8 & 2.9 & 2.9 & 2.8 & 2.9 & 2.9 \\
\hline & $\operatorname{Strain}(\mathrm{mm} / \mathrm{mm})$ & $1.2 \mathrm{e}^{-3}$ & $1.2 \mathrm{e}^{-3}$ & $1.2 \mathrm{e}^{-3}$ & $9.0 \mathrm{e}^{-4}$ & $9.2 \mathrm{e}^{-4}$ & $9.3 \mathrm{e}^{-4}$ & $7.0 \mathrm{e}^{-4}$ & $7.1 \mathrm{e}^{-4}$ & $7.7 \mathrm{e}^{-4}$ \\
\hline & Total def.(mm) & $3.9 \mathrm{e}^{-4}$ & $2.2 \mathrm{e}^{-3}$ & $3.5 \mathrm{e}^{-3}$ & $2.9 \mathrm{e}^{-4}$ & $1.7 \mathrm{e}^{-3}$ & $2.7 \mathrm{e}^{-3}$ & $2.3 \mathrm{e}^{-4}$ & $1.3 \mathrm{e}^{-3}$ & $2.1 \mathrm{e}^{-3}$ \\
\hline & Stress (MPa) & 5.0 & 6.8 & 6.6 & 5.0 & 6.8 & 6.6 & 5.0 & 6.8 & 6.6 \\
\hline & $\operatorname{Strain}(\mathrm{mm} / \mathrm{mm})$ & $2.1 \mathrm{e}^{-3}$ & $2.8 \mathrm{e}^{-3}$ & $2.8 \mathrm{e}^{-3}$ & $1.6 \mathrm{e}^{-3}$ & $2.1 \mathrm{e}^{-3}$ & $2.1 \mathrm{e}^{-3}$ & $1.3 \mathrm{e}^{-3}$ & $1.7 \mathrm{e}^{-3}$ & $1.7 \mathrm{e}^{-3}$ \\
\hline
\end{tabular}
The constraint condition of the model is firstly that the base of single MN fixed with $400 \mu \mathrm{m}$ diameter and $100 \mu \mathrm{m}$ height thin plate, then the MN without fixing plate.

Table 2. Stress, Strain, Total deformation results done by ANSIS (with fixing plate) 
Three different geometries of MNs were designed to explore the mechanics regularities of MNs, three kinds of material were used. Putting an axial pressure $3.183 \mathrm{MPa}$, also the same pressure with $30^{\circ}$ piercing angle on the tip of the MN, the results are shown in Table 2 , and Fig. 2.
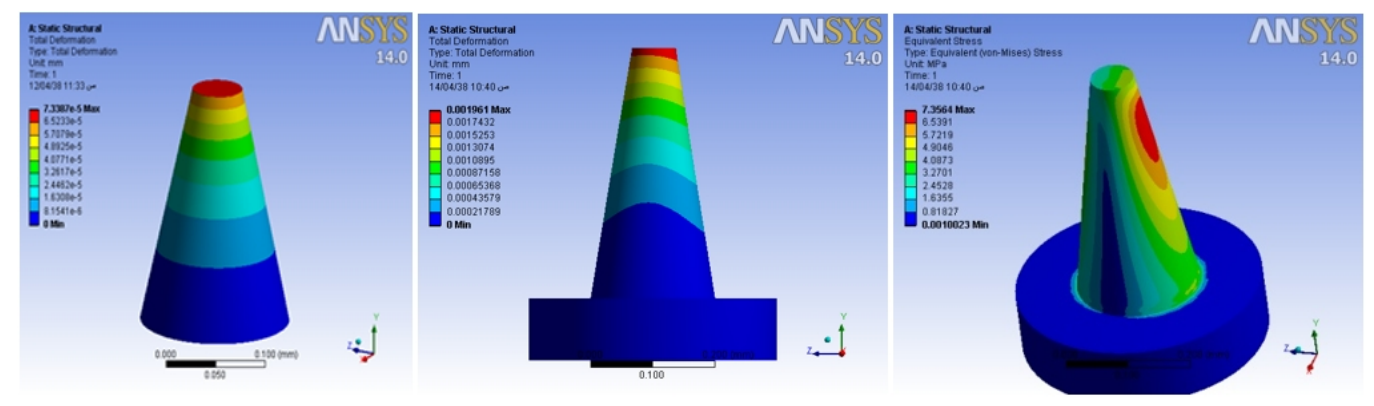

Fig. 2. FEM simulation analysis for the stress and total deformation of PC, PMMA and PLA MN

\section{Conclusions}

In this research, first $\mathrm{MN}$ model was designed and the main forces that a $\mathrm{MN}$ suffered using MATLAB was calculated, and stresses using an ANSYS was analysed for three different polymer materials PMMA, PC and PLA. The results shows that all suggested polymer materials applied for this designed model can satisfy the requirements for safe use of MNs.

\section{References}

[1] Ganesan, Adarsh Venkataraman, Hardeep Kumar, S. Swaminathan, K. K. Singh, Reenu Anne Joy, Neeru Sood, Trupti Gokhale, and R. K. Mittal. "Analysis of MEMS-Based Microneedles for Blood Monitoring." Bionanoscience 4, no. 2 (2014): 128-135.

[2] Kaushik, Shilpa, Allen H. Hord, Donald D. Denson, Devin V. McAllister, Sudhasinee Smitra, Mark G. Allen, and Mark R. Prausnitz. "Lack of pain associated with microfabricated microneedles." Anesthesia \& Analgesia 92, no. 2 (2001): 502-504.

[3] Prausnitz, Mark R., and Robert Langer. "Transdermal drug delivery." Nature biotechnology 26, no. 11 (2008): 1261.

[4] Prausnitz, Mark R. "Analysis: overcoming skin's barrier: the search for effective and user-friendly drug delivery." Diabetes technology \& therapeutics 3, no. 2 (2001): 233-236.

[5] Fukushima, Keizo, Takenao Yamazaki, Ryo Hasegawa, Yukako Ito, Nobuyuki Sugioka, and Kanji Takada. "Pharmacokinetic and pharmacodynamic evaluation of insulin dissolving MNs in dogs." Diabetes technology \& therapeutics 12, no. 6 (2010): 465-474.

[6] Kim, Yeu-Chun, Jung-Hwan Park, and Mark R. Prausnitz. "Microneedles for drug and vaccine delivery." Advanced drug delivery reviews 64, no. 14 (2012): 1547-1568.

[7] Wilson, Carol J., and Patricia A. Beck. "Fracture testing of bulk silicon microcantilever beams subjected to a side load." Journal of Microelectromechanical Systems 5, no. 3 (1996): 142-150.

[8] Bodhale, D. W., A. Nisar, and N. Afzulpurkar. "Design, Fabrication and analysis of silicon MNs for TDD applications." In The Third, International Conference on the Development of Biomedical Engineering in Vietnam, pp. 84-89. Springer, Berlin, Heidelberg, 2010. 